Carter of the University of Connecticut in Storrs had invited Pennycook to contribute a chapter to a future reference book on electron microscopy, with a possibility to share royalties. Williams did not declare the interaction. Oak Ridge claims that when it found out - well after the investigation had been completed - its lawyer said that there was no conflict of interest.

\section{Web alliance speeds up communication with Africa}

African researchers have just been connected by a high-speed Internet link to Europe's research network, GÉANT2. The deal has been struck between GÉANT2 and UbuntuNet, an alliance of African national research networks created in 2005 . It will speed up communications and data transfer not only between African scientists and the estimated 30 million GÉANT2 users in 34 European countries, but also with scientists worldwide - through GÉANT2's connections with the United States and other research networks around the world.

UbuntuNet is not related to Ubuntu, the popular free version of the Linux operating system. But both namesakes help bring affordable quality information technology to African scientists.

\section{Review article retracted amid plagiarism claims}

A review article written by a rheumatologist at Harvard Medical School in Boston, Massachusetts, has been retracted after the journal, Best Practice \& Research Clinical Rheumatology, learned that more than half of the paper may have been plagiarized.

The 2004 article, by Lee Simon (Best

Pract. Res. Clin. Rheumatol. 18, 507-538;
2004), was manually checked after surfacing in an automated trawl through 7 million biomedical abstracts for possible plagiarism (see Nature 451, 397-399; 2008). The retraction was announced on 29 January.

Harvard Medical School has formed a committee to review the matter but has not launched an official investigation, says spokesman David Cameron. Simon declined to comment, saying only: "I'm very sorry that I've been so targeted for something like a review article."

\title{
Probe catches glimpse of surface troughs on Mercury
}

Down on the floor of the immense Caloris Basin - an impact crater at least 1,300 kilometres wide that scars the surface of the planet Mercury

- lies a set of radial troughs nicknamed 'the spider' (right). This image is one of many sent back by NASA's MESSENGER spacecraft during its 14 January flyby, the first of three planned before it settles into orbit around Mercury in 2011. Project scientists think the troughs in

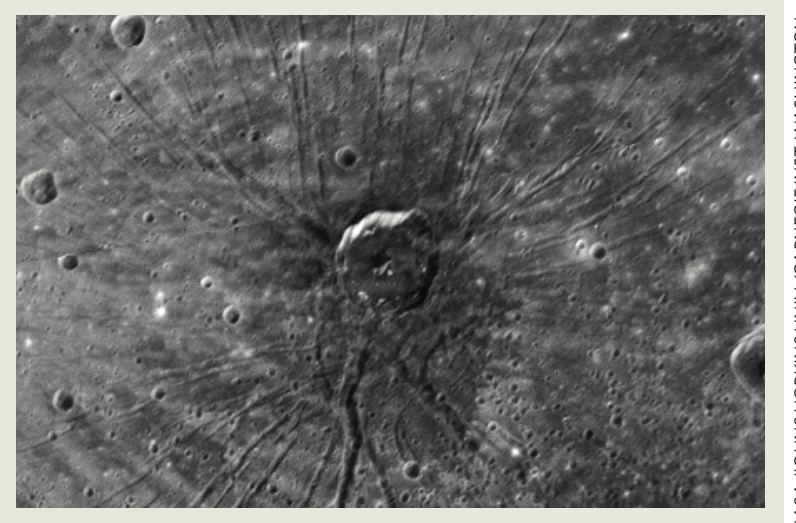

the spider formed as material in the floor of the Caloris Basin pulled apart. A smaller impact crater near the centre may also have contributed to trough formation. 